\title{
A New Combination Method of Temperature and Vehicle Load Effects
}

\section{Based on SHM Data}

\author{
Wang Zijiang ${ }^{1, a}$, Hu Rongpan ${ }^{1, b}$ and Li Shunlong ${ }^{2, c}$ \\ ${ }^{1}$ Key Laboratory of Advanced Material \& Intelligent Control Technology on Transportation Safety, \\ Harbin Institute of Technology, Harbin, China \\ ${ }^{2}$ School of Transportation Science and Engineering, Harbin Institute of Technology, 150090, \\ Harbin, China \\ ªwangzijian1987@163.com, 'brongpan.hu@gmail.com, 'Pishunlong@hit.edu.cn
}

Keywords: Load Effects Combination, Stochastic Process, Structural Health Monitoring.

\begin{abstract}
When more than one load act on a structure, the combination of the load effects should be considered, especially for which are variant in time and in space. A new method to calculate the combination of two different loads effects as well as to predict the extreme value distributions in the subsequent service life is proposed. The loads discussed in this paper are two main loads acting on the cable-stayed bridge: the temperature and vehicle load, which can be modeled as rectangular pulse process and filtered Poisson process respectively. Firstly, truncated distributions of the two load effects are depicted using the monitoring data of Nanjing 3rd Yangtze River Bridge. The generalized Pareto distribution fits well to the upper tails of the vehicle load effect and the mean values of the temperature load effect in an hour follows a two weighted normal distribution. Then the combination of the two load effects as well as the prediction of extreme values in the subsequent service life can be calculated. In the end, the results obtained through the proposed method are compared with the observed value of the bridge.
\end{abstract}

\section{Introduction}

Load effects are important concerns in structure design and structural safety analysis. As a structure is usually subjected to several different kinds of loads simultaneously, the combination of the load effects should be considered. Structural loads, which vary in time (e.g., wind load, vehicle load, seismic load, temperature load, etc.) and in space are usually modeled as stochastic process. To find a method to combine these stochastic loads accurately and conveniently has always been a concern for many scholars.

Turkstra's rule commonly adopted to estimate the combination of several stochastic loads because of its simplicity [1]. However, the estimation is non-conservative as it ignores the possibility that different load processes might attain the maximum values simultaneously [2]. Ferry-Borges and Castanheta method is simply and widely used. It ignores the short-term temporal fluctuation and correlation [3]. Wen proposed load coincidence method based on the model of Poisson process [4]. The exceed probability of the combination can be estimated according to the incidence ratio of load combination. Larrabee and Cornell [5] developed a point crossing formula applicable to the linear combination of stable and independent stochastic load processes. Yasuhiro Mori and Takahiro Kato [6] developed a theoretical expression of the combination of a Poisson square wave process and a Poisson impulse process. 
This paper presents the theoretical expression of the cumulative distribution function (cdf) of the combination of two stochastic load effects commonly concerned in bridges. These two loads refer to the temperature load and the vehicle load which cause the deck stress of the long span cable-stayed bridges. The real-time data of these load effects, collected by the structural health monitoring system which is permanently installed on Nanjing 3rd Yangtze River Bridge will be used for the analysis of the next section.

\section{Identification of vehicle load effect and temperature load effect}

As temperature load effect varies significantly in different seasons, the monitoring data are expected to include every month to guarantee an accurate evaluation of the temperature load effects distribution properties. The deck stress time history in 278 days collected by the No.13 sensor during 2006.1 2011.12 has been selected for the analysis in the following section.

Moving average method obtains a series of average values to reflect the trend of the time-variant sequence, as shown in Fig. 1.

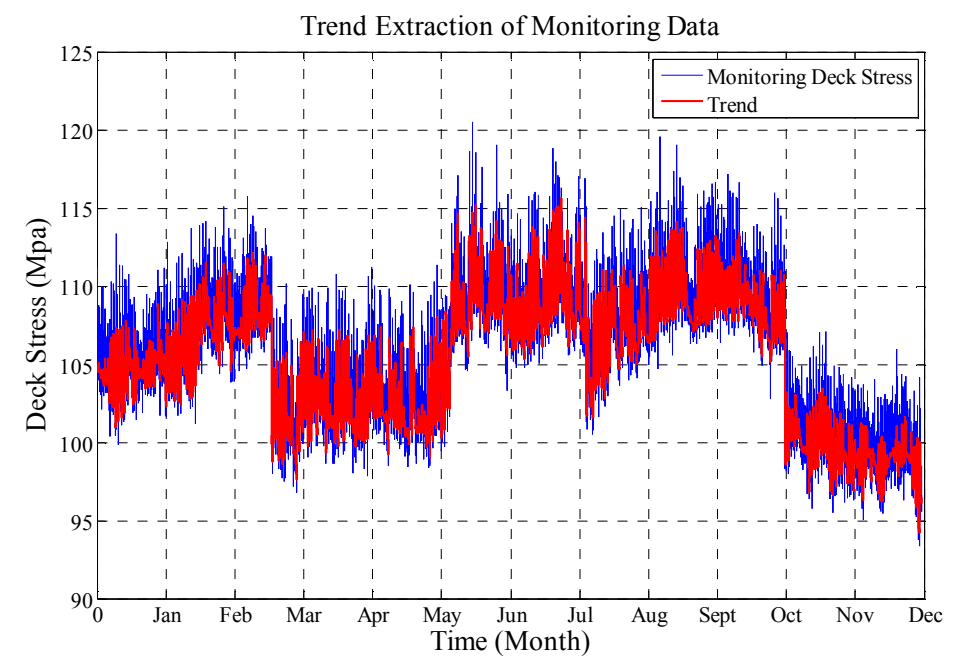

Fig. 1 Trend extraction from deck stress time history

Removing the mean from the trend, the deck stress time history can be categorized into three parts: vehicle stress (shown in Fig. 2), temperature stress (shown in Fig. 3) and constant load stress (105.3471 MPa).

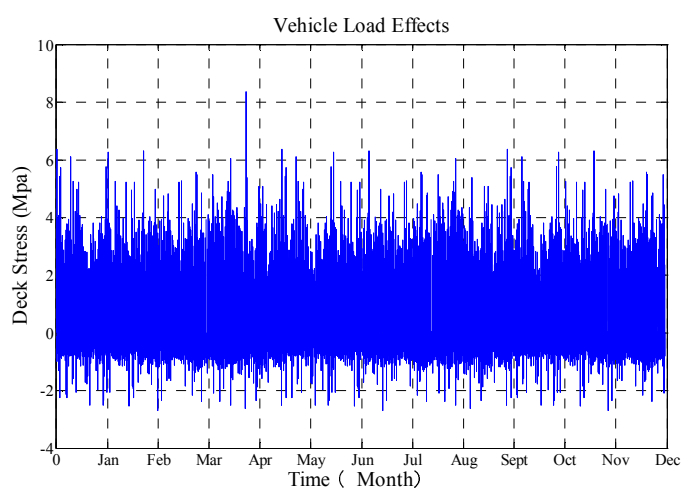

Fig. 2 Vehicle stress time history

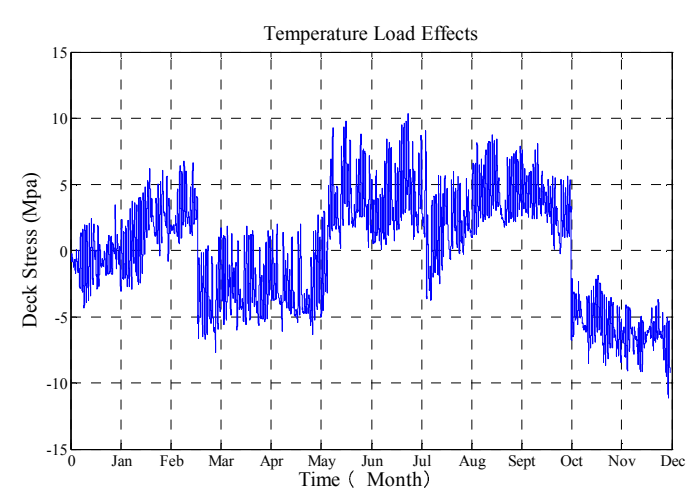

Fig. 3 Temperature stress time history 
In the extreme value distribution estimation, the tail part of the stress would be points of interest. generalized Pareto distribution is employed to fit the upper tail of vehicle load effects, where the threshold is chosen as $1 \mathrm{MPa}$.

$$
F_{L}(x)=1-\left(1-\frac{0.0807(x-1)}{0.8640}\right)^{1 / 0.0807} \text {. }
$$

As the vehicle load effect can be modeled as filtered Poisson process, cdf of the extreme value distribution can be expressed as:

$$
F_{L \max }(x)=\exp \left\{-\lambda T_{s}\left(1-F_{L}(x)\right)\right\} .
$$

where $\lambda$ means the parameter of the Poisson process, $\mathrm{T}_{\mathrm{s}}$ denotes the subsequent service life and $\mathrm{F}_{\mathrm{L}}(\mathrm{x})$ represents the cdf of truncated vehicle load effect.

Monte-Carlo simulation provides an effective way to obtain the pdf of different service life. Statistical results indicate that the pdf of hourly mean temperature load effect can be fitted by two weighted normal distribution. The fitting equation can be expressed as:

$$
F_{T(x)}=0.6369 \Phi\left(\frac{x-2.463}{1.3756}\right)+0.3631 \Phi\left(\frac{x+4.671}{1.1861}\right) \text {. }
$$

\section{Combination of two stochastic load effects}

The vehicle load effect can be described approximately as filtered Poisson process as shown in Fig. 4 and the temperature load effect can be modeled as rectangular pulse process with the duration time $\mu_{\mathrm{d}}$ being 3600 seconds as shown in Fig. 5 .

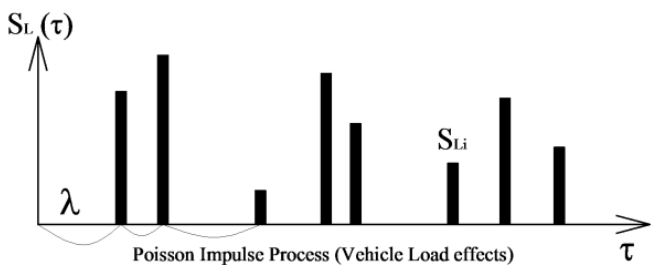

Fig. 4 Filtered Poisson process

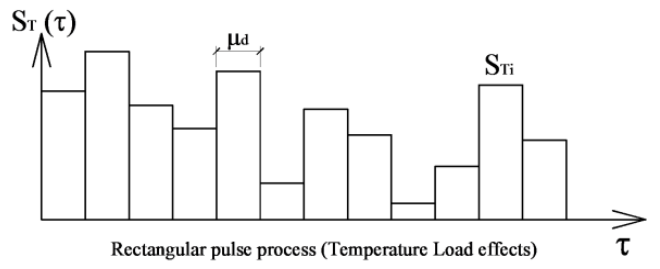

Fig. 5 Rectangular pulse process

The cdf of the maximum intensity of $Z_{d \max }$ in the subsequent service life $T_{\mathrm{s}}$ can be expressed as (4) $[6]$ :

$$
F_{Z d \max }(z)=\left[\int_{0}^{\infty} \exp \left\{-\lambda \mu_{d}\left[1-F_{S_{L}}\left(z-z_{T}\right)\right]\right\} f_{z_{T}}\left(z_{T}\right) d z_{T}\right]^{T_{s}} .
$$

Numerical integration is applied to calculate the values of $\mathrm{F}_{\mathrm{zdmax}}(\mathrm{z})$ in different subsequent service life and the curves are plotted in Fig. 6. The pdf (shown in Fig. 7) of daily extreme value is also calculated by Monte-Carlo simulation from the corresponding curve in Fig. 6.

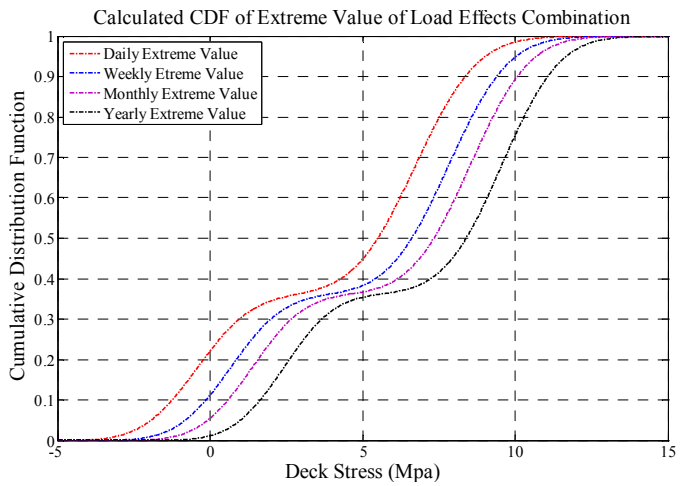

Fig. 6 Calculated cdf of combination

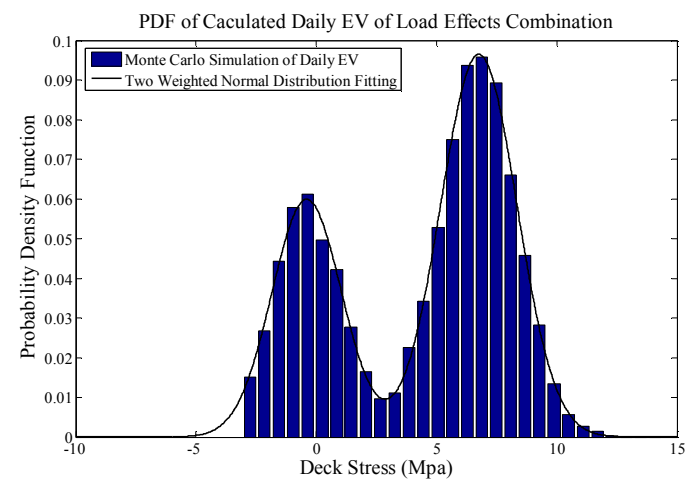

Fig. 7 Calculated pdf of combination 
To certify the effectiveness of this combination method, a comparison has been made between the proposed method and other rules(e.g. the Turkstra's rule and the Borges' rule). The calculated pdfs of hourly maximum load effect are shown in Fig.8.

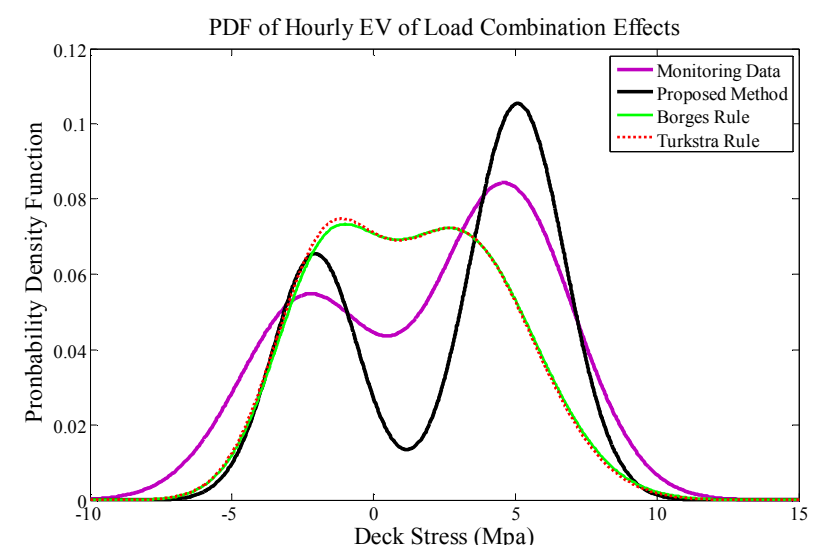

Fig. 8 Comparison between different combination methods

The overlapping areas between calculated pdf curves and the empirical pdf curve are calculated and shown in Table 1.The result indicates the validity of the proposed combination method .

Table 1 Result of calculated overlapping areas

\begin{tabular}{|c|c|}
\hline Different combination method & Overlapping area \\
\hline Turkstra's rule & 0.7100 \\
\hline Borges' rule & 0.7107 \\
\hline Proposed method & 0.7275 \\
\hline
\end{tabular}

\section{Conclusion}

This paper reviews the development of the combination rules of several stochastic loads and proposes a method of combining two stochastic loads which can be modeled as rectangular process and filtered Poisson impulse process respectively. The analysis of the vehicle and temperature load effects is based on the real-time data collected through the SHM system which accurately reflect the real condition of the bridge. To describe the combination of load effects, the truncated distribution of two stochastic processes is discussed firstly. The generalized Pareto distribution is applied to fit the upper tail of the vehicle load effect while the temperature load effect follows two weighted normal distribution. Then numerical integration is applied to calculate the cdf of combination and the prediction of maximum stress can be obtained from calculated cdf by means of the Monte-Carlo simulation. In the end, a comparison is made between the proposed method and other combination rules. Result of the comparison proves the validity and accuracy of the proposed method.

\section{References}

[1] Turkstra C.J., Theory of structural design decision, Solid Mechanics Division, Study NO. 2, University of Waterloo, Waterloo, Ont, 1970.

[2] Howard T. Pearce, Y. K. Wen, Stochastic Combination of Load Effects, J. Struct. Eng, ASCE, 110 (1984)1613-1629.

[3] J. Ferry-Borges, M. Castenheta, Structural Safety, Laboratoria Nacional de Engenhera Civil, Lisbon, 1971. 
[4] Wen Y.K., Statistical combination of extreme loads, J. Struct. Div, ASCE, 103(1977)1079-1093.

[5] Larrabee R.D., Cornell C.A. Combination of various load processes, J. Struct. Div, ASCE, 107(1981)223-239.

[6] Yasuhiro Mori, Takahiro Kato, Kazuko Murai, Probability models of combinations of stochastic loads for limit state design, Structural Safety 25 (2003) 69-97. 